\title{
CCN proteins, microenvironment, communication and signaling: why did we need a new journal?
}

\author{
Annick Perbal • Bernard Perbal
}

Received: 8 April 2007 / Accepted: 9 April 2007 / Published online: 25 May 2007

(C) The International CCN Society 2007

Keywords Signalling . Communication .

$\mathrm{CCN}$ family of proteins $\cdot \mathrm{CCN} 3 \cdot$ Microenvironment .

Intercellular $\cdot$ Intracellular

The six proteins of the "CCN family" constitute a new group of cell proliferation and differentiation regulators that play key roles at various stages of normal and pathological development (Perbal and Takigawa 2005). The wide range of biological properties that have been attributed to the $\mathrm{CCN}$ proteins stems from their conserved multimodular achitecture, with four structural modules that share partial identity with four other large families of regulatory proteins (Bork 1993; Perbal 2001, 2002).

The presence of a signal peptide at the amino terminus of all $\mathrm{CCN}$ proteins suggested that they were secreted. Indeed, $\mathrm{CCN}$ proteins were detected outside of cells, at the cell membrane, in the culture medium, or in the extracellular matrix (Chevalier et al. 1998; Yang and Lau 1991; Kleer et al. 2004; Lechner et al. 2000; Perbal 2001). As a consequence, attention was first drawn to the potential effects biological effects of CCN1-6 as ligands for "classical" type of receptors and later as matricellular proteins (Bornstein 1995).

It is now widely accepted that $\mathrm{CCN}$ proteins are unlikely to play a structural role in the extracellular matrix (ECM), but rather play essential regulatory functions in matrixrelated signaling through their interactions with other components of the ECM (Leask and Abraham 2006).

A. Perbal $\cdot$ B. Perbal $(\bowtie)$

Laboratoire d'Oncologie Virale et Moléculaire,

Université Paris 7 D. Diderot, Paris, France

e-mail: perbal@ccr.jussieu.fr
The connection that was made with integrin signaling (Lau and Lam 2005) provided a solid ground for the biological effects of CCN proteins on cell biology ex vivo and action on angiogenesis and skeletal development in vivo. The involvement of integrins in fundamental aspects of signaling is well documented and there is no doubt that the various levels at which they act as receptor and transmitter of regulatory signals may account for the wide range of $\mathrm{CCN}$ protein functions.

From early sequence analyses, one could easily predict that these four modules would confer on the $\mathrm{CCN}$ proteins a remarkable potential to interact with numerous partners and act through various signaling pathways (Perbal 2001). Indeed, experiments performed with isolated cellular systems established that $\mathrm{CCN}$ proteins were involved in several regulatory cascades including MAPK and AKT pathways.

The pleiotropic functions of $\mathrm{CCN}$ proteins have emerged in quite unforeseen directions with reports establishing that $\mathrm{CCN}$ proteins also physically interact with an increasing number of receptors and ligands, in the ECM, biological fluids, and even within the cell cytoplasm and nucleus (Perbal 2001, 2004). Structural considerations had previously led us to propose that the biological functions of CCN proteins might result from combinatorial events, with $\mathrm{CCN}$ proteins governing or participating in the formation of multimolecular complexes whose constitution would be critical for CCN-driven signaling (Perbal 2001). The multiplicity of potential partners and subcellular sites of action makes CCN1-6 a fascinating class of regulatory proteins (Fig. 1).

Considering that the developmental expression patterns of $\mathrm{CCN}$ proteins result from a tight spatio-temporal regulation, and that these six structurally related $\mathrm{CCN}$ proteins play non-redundant functions, it is not so 
Inter-cellular signaling and medium sensing

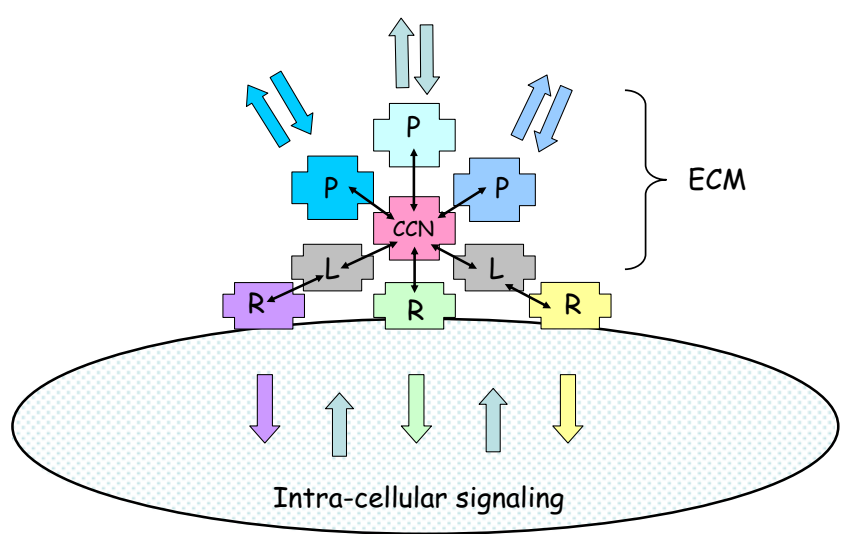

Fig. 1 Schematic representation of the multiple types of interactions in which $\mathrm{CCN}$ proteins may be involved for signaling cell functions. $C C N$ CCN proteins, $L$ ligands of receptors, $R$ receptor, $E C M$ extracellular matrix, $P$ potential partners in the extracellular matrix

surprising in the end that they are involved in such a wide variety of regulatory circuitry.

However, it is also obvious that studying the functions of such proteins in isolated systems might be considering only one side of the coin, since most of the ex vivo studies deal with systems that lack matrix components and microenvironemental factors which are known to be of prime importance in normal homeostasis.

Microenvironment modifications including matrix remodeling are the key to the intercellular signaling that is required among many other situations, for balanced morphogenesis during development, for tissue remodeling after healing, and for cell invasion in tumor metastasis.

It is essential to identify those macromolecules which are involved in the coordination of high level signaling pathways that govern the birth, life and death of cells in their normal three dimensional environment.

We believe that $\mathrm{CCN}$ proteins are key players in these integrated processes. We believe that the apparent complexity and contradictions that we see in the biological functions of $\mathrm{CCN}$ proteins stem from our relative ignorance of the spatial control of communication.

Studies performed in vivo with recombinant mice (Mo et al. 2002; Ivkovic et al. 2003) should help us to clarify the functions of $\mathrm{CCN}$ proteins during normal development but might not permit us to address specific pathological situations in which alterations of $\mathrm{CCN}$ proteins production or structure have been associated (Planque and Perbal 2003). There is a need for a wider use of ex vivo systems that can mimic the cell microenvironment. Along this line, it is worth noting that the use of skin reconstructs permitted establishment that CCN3 is involved in the 3D fate of melanocytes (Fukunaga-Kalabis et al. 2006). Such approaches are extremely promising and should permit the study of the role of $\mathrm{CCN}$ proteins in a more "biological" context.
These brief considerations highlight the place of CCN proteins as central actors in the complex hub of regulatory signals that govern life and death. There is no doubt that intercellular signaling calls for involvement of many other types of coordinators. However, we believe that the CCN proteins constitute a very attractive family of proteins that may well become the focus of many experiments aimed at understanding spatial signaling and communication.

The lack of a journal devoted to these interdisciplinary approaches had previously led us to create the CCS electronic journal published by BioMed Central. With the development of the $\mathrm{CCN}$ field and the many ramifications that $\mathrm{CCN}$ show with several other fundamental regulatory pathways, we have felt that it was time to proceed further with the launching of the Journal of Cell Communication and Signaling (JCCS), JCCS is the official organ journal of the International CCN Society (ICCNS). It replaces the previous CCS journal hosted by BMC. Although it is the official organ of the ICCNS, JCCS should serve as a forum for the dissemination of current knowledge and information dealing with all aspects of cell-cell, cell-matrix communication, and intracellular signaling.

We have adopted an interdisciplinary approach, reflecting the breadth and depth of the membership of the ICCNS and the functional relationships of the $\mathrm{CCN}$ field with others. As such, JCCS covers identification and function of receptors, ligands, and other biomolecules participating in the outward and inward cellular signalling pathways in normal and pathological conditions.

Cell-cell, cell-matrix, cell-environment, and cell-microorganisms interactions modulate or even override programmed patterns of cell behaviour. In this respect, signalling is critical for normal cell growth and development, and abnormal signalling pathways have been associated with several human diseases. JCCS intends to publish reports dealing with all these aspects, incorporating morphological, biochemical, molecular and physiological approaches.

JCCS especially welcomes the publication of studies reporting innovative in vitro models that facilitate investigations of signaling networks and cell behaviour.

The success of any journal depends to a great extent on the quality of its published articles. It also depends on the scientific curiosity of its readers. We do hope that JCCS will provide the scientific community with a new vehicle for dissemination of good science and for the coordination of work that should eventually lead to better knowledge of integrated signaling and thereby open new avenues for molecular medicine.

Acknowledgements We are greatful to Dr. H. Yeger for critical reading of the manuscript. Financial support from European Commission (grant number: PROTHETS LSHC-CT-2004-50303306). 


\section{References}

Bork P (1993) The modular architecture of a new family of growth regulators related to connective tissue growth factor. FEBS Lett 327:125-130

Bornstein P (1995) Diversity of function is inherent in matricellular proteins: an appraisal of thrombospondin 1. J Cell Biol 130:503506

Chevalier G, Yeger H, Martinerie C, Laurent M, Alami J, Schofield P, Perbal B (1998) novH: differential expression in developing kidney and Wilms' tumours. Am J Pathol 152:1563-1575

Fukunaga-Kalabis M, Martinez G, Liu ZJ, Kalabis J, Mrass P, Weninger W, Firth SM, Planque N, Perbal B, Herlyn M (2006) $\mathrm{CCN} 3$ controls 3D spatial localization of melanocytes in the human skin through DDR1. J Cell Biol 175:563-569

Ivkovic S, Yoon BS, Popoff SN, Safadi FF, Libuda DE, Stephenson RC, Daluiski A, Lyons KM (2003) Connective tissue growth factor coordinates chondrogenesis and angiogenesis during skeletal development. Development 130:2779-2791

Kleer CG, Zhang Y, Pan Q, Merajver SD (2004) WISP3 (CCN6) is a secreted tumor-suppressor protein that modulates IGF signaling in inflammatory breast cancer. Neoplasia 6:179-185

Lau L, Lam S (2005) Integrin-mediated CCN functions. In: Perbal B, Takigawa M (eds) The CCN proteins: a new family of cell growth and differentiation regulators. Imperial College Press, London, pp 61-79
Leask A, Abraham DJ (2006) All in the CCN family: essential matricellular signaling modulators emerge from the bunker. $\mathrm{J}$ Cell Sci 119:4803-4810

Lechner A, Schutze N, Siggelkow H, Seufert J, Jakob F (2000) The immediate early gene product hCYR61 localizes to the secretory pathway in human osteoblasts. Bone 1:53-60 (erratum in: Bone 2000 October 27(4):569)

Mo FE, Muntean AG, Chen CC, Stolz DB, Watkins SC, Lau LF (2002) CYR61 (CCN1) is essential for placental development and vascular integrity. Mol Cell Biol 22:8709-8720

Perbal B (2001) NOV (nephroblastoma overexpressed) and the CCN family of genes: structural and functional issues. Mol Pathol 54:57-79

Perbal B (2002) Les protéines CCN: quand multimodulaire rime avec multifonctionnel. Médecine/Sciences 18:745-756

Perbal B (2004) CCN proteins: multifunctional signalling regulators. Lancet 363:62-64

Perbal B, Takigawa M (2005) The CCN family of proteins: an overview. In: Perbal B, Takigawa M (eds). The CCN proteins: a new family of cell growth and differentiation regulators. Imperial College Press, London, pp 1-18

Planque N, Perbal B (2003) A structural approach to the role of CCN (CYR61/CTGF/NOV) proteins in tumourigenesis. Cancer Cell Int 3(1):15

Yang GP, Lau LF (1991) Cyr61, product of a growth factor inducible immediate early gene, is associated with the extracellular matrix and the cell surface. Cell Growth Differ 2:351-357 